\title{
English Language Proficiency and Geographical Proximity to a Safety Net Clinic as a Predictor of Health Care Access
}

\author{
Kristina M. Cordasco $\cdot$ Ninez A. Ponce • \\ Melissa S. Gatchell $\cdot$ Brandon Traudt • \\ José J. Escarce
}

Published online: 18 December 2010

(C) The Author(s) 2010. This article is published with open access at Springerlink.com

\begin{abstract}
Studies suggest that proximity to a safety net clinic (SNC) promotes access to care among the uninsured. Distance-based barriers to care may be greater for people with limited English proficiency (LEP), compared to those who are English proficient (EP), but this has not been explored. We assessed the relationship between distance to the nearest SNC and access in non-rural uninsured adults in California, and examined whether this relationship differs by language proficiency. Using the 2005 California Health Interview Survey and a list we compiled of California's SNCs, we calculated distance between uninsured interviewee residence and the exact address of the nearest SNC. Using multivariate regression to adjust for other relevant
\end{abstract}

This paper was presented at The Society of General Internal Medicine Meeting in Pittsburgh, PA, April 11, 2008 as well as at the Academy Health meeting in Washington, DC, June 8th, 2008.

\section{K. M. Cordasco $(\square)$}

The Greater Los Angeles VA Healthcare System,

University of California, 11301 Wilshire Blvd (111G),

Los Angeles, CA 90073, USA

e-mail: kcordasco@mednet.ucla.edu

\section{K. M. Cordasco $~$ J. J. Escarce}

Department of Medicine, School of Medicine,

The University of California, Los Angeles, CA, USA

\section{J. J. Escarce}

e-mail: jescarce@mednet.ucla.edu

\section{N. A. Ponce - M. S. Gatchell - B. Traudt}

The Center for Health Policy Research, School of Public Health, The University of California, 31-254B CHS, BOX 951772,

Los Angeles, CA 90095-1772, USA

e-mail: nponce@ucla.edu

M. S. Gatchell

e-mail: gatchell@ucla.edu characteristics, we examined associations between this distance and interviewee's probability of having a usual source of health care (USOC) and having visited a physician in the prior 12 months. To examine differences by language proficiency, we included interactions between distance and language proficiency. Uninsured LEP adults living within 2 miles of a SNC were $9.3 \%$ less likely than their EP counterparts to have a USOC $(P=0.046)$. Further, distance to the nearest SNC was inversely associated with the probability of having a USOC among LEP, but not among EP; consequently, the difference between LEP and $\mathrm{EP}$ in the probability of having a USOC widened with increasing distance to the nearest SNC. There was no difference between LEP and EP adults living within 2 miles of a SNC in likelihood of having a physician visit; however, as with USOC, distance to the nearest SNC was inversely associated with the probability of having a physician visit

\author{
B. Traudt \\ e-mail: Brandon.C.Traudt@kp.org \\ K. M. Cordasco · J. J. Escarce \\ The RAND Corporation, Santa Monica, CA, USA \\ B. Traudt \\ Department of Clinical Analysis, Southern California \\ Permanente Medical Group, Pasadena, CA 91188, USA \\ M. S. Gatchell \\ University of California, 21-254B CHS, Box 951772, \\ Los Angeles, CA 90095, USA \\ J. J. Escarce \\ UCLA Division of General Internal Medicine \\ and Health Services Research, 911 Broxton, \\ BOX 951736, Los Angeles, CA 90095-1736, USA
}


among LEP but not EP. The effect sizes diminished, but remained significant, when we included county fixed effects in the models. Having LEP is a barrier to health care access, which compounds when combined with increased distance to the nearest SNC, among uninsured adults. Future studies should explore potential mechanisms so that appropriate interventions can be implemented.

Keywords Linguistic disparities - Access to care . Uninsured · Health care safety net - Geographic analysis . Secondary analysis

\section{Background}

On average, people who lack health insurance use less health care and have worse health outcomes than those with health insurance. In particular, the uninsured are less likely to have a usual source of health care, have had any physician visit in the prior year, obtain preventive health care services, and seek care for urgent and chronic conditions [1-5]. Multiple studies suggest that the uninsured have worse overall health and higher mortality rates [6]. People without insurance often access primary health care through the core healthcare safety net-a patchwork of providers predominantly composed of publicly-supported hospitals and safety net clinics (SNCs), such as community health centers $[7,8]$. The structure of the safety net varies widely across communities, most commonly reflecting the community's finances, politics, history and needs [7].

Previous studies suggest that the structure of the health care safety net may affect health care access and utilization, with geographic proximity to a SNC promoting better access among uninsured adults [9, 10]. Hadley and Cunningham, using the 1998-1999 Community Tracking Study (CTS), showed that proximity of residence to the nearest SNC is positively associated with having a usual source of care [9]. Similarly, Gresenz and colleagues, using the Medical Expenditure Panel Survey (MEPS), found a positive association between health care utilization and proximity of residence to the nearest SNC for rural residents. Among urban residents, greater distance to the nearest public hospital was associated with more emergency department visits, possibly due to substitution for office-based visits [10]. In both studies, the authors concluded that expanding the health care safety net so that more uninsured adults live close to a SNC could improve healthcare access to ambulatory care [9, 10].

With the exception of differences between rural and urban residents, these studies have not examined whether there are subpopulations among the uninsured that are at higher risk for having distance-based disparities in access and utilization. Probst and colleagues [11], using the 2001
US National Household Travel Survey, found that urban and rural African-Americans, compared to Whites, had significantly more trips of $30 \mathrm{~min}$ or longer to access healthcare. The authors suggest that this finding may be attributable to a higher proportion of African-Americans using public transportation and having other characteristics that contribute to higher travel time burdens [11].

Another subpopulation that may be at increased risk of distance-based disparities in healthcare access and utilization is those with limited English proficiency (LEP). It is well established that people with LEP have less access to and lower use of healthcare [12, 13]. However, a recent study of parents of Latino children, many of whom had LEP, suggests that barriers to care in this population go beyond language, and that the influence of these barriers may be exacerbated by distance to providers. Specifically, parents reported that lack of transportation to a health clinic, and the time accessing care, were prevalent and significant obstacles to obtaining medical care for their children [14]. Studies of how geographic distance may differentially affect subpopulations among the uninsured could guide the targeting of interventions to diminish disparities.

In this study, we examine the relationship between distance to the nearest SNC and health care access among urban and suburban uninsured adults in California and we assess whether the effects of distance to the nearest SNC on access differ between uninsured people with LEP and those who are English proficient (EP).

\section{Data and Methods}

\section{Data Collection and Sources}

\section{California Health Interview Survey}

We used the confidential data files of the 2005 California Health Interview Survey (CHIS) to obtain a sample of uninsured Californians. The 2005 CHIS was a randomdigit dial telephone survey, stratified by 41 county or county groups, and the random sample was augmented by Vietnamese and Korean surname list samples. Interviews were conducted in English, Spanish, Cantonese, Mandarin, Vietnamese and Korean. Sample weights are utilized to adjust for list sample augmentation, exclusion of households without landlines, and nonresponse bias so that it is representative of noninstitutionalized Californians living in households. The major survey domains of CHIS 2005 included detailed sociodemographic characteristics, including language use and proficiency; overall health and health conditions; health care access and utilization; and health behaviors. 
The overall response rate for CHIS 2005 was $27 \%$, yielding 43,020 adult surveys. An analysis of CHIS 2005 nonrespondents showed that they had having similar neighborhood characteristics as respondents [15]. Among respondents, 3,759 (9\%) were uninsured for the entirety of the prior 12 months. Of these, we included in our sample those who were not pregnant, lived in urban or suburban neighborhoods, and provided their exact address or nearest cross street such that we could determine the latitude and longitude of their residence. We excluded subjects with rural residence, based on a CHIS 2005 variable that categorized neighborhoods as "urban," "second city," "suburban," or "rural." We additionally excluded 27 persons who were classified as urban but lived 10 or more miles from the nearest SNC, as they were few and their communities of residence were not typically urban. Our final study sample included 2,740 subjects.

\section{Safety Net Clinics}

To compile an initial list of California's safety net clinics, we visited the websites of key California health care safety net organizations, as listed in the California Healthcare Foundation's 2005 report, "California's Safety-Net Clinics: A Primer" [16]. We augmented this list with governmental listings of all California Federally Qualified Health Centers (FQHCs), FQHC look-alikes, community health clinics, free clinics, rural health clinics and county clinics [17, 18].

Between July 2007 and January 2008, we telephoned each clinic to administer a 3-min survey, verifying the exact address, clinical services provided, insurance status of patient population, and payment policies. If the clinic phone number originally obtained was incorrect, we utilized internet search engines, directory assistance and calling nearby health care service providers to find alternate numbers. We excluded a clinic only if we were able to verify its nonexistence through being told such by a parent, partner, or nearby organization. For the analyses presented in this paper, we selected from this list clinics that provided uninsured adults with general medical services for free or at a reduced fee, including a "sliding-scale" fee based on income.

\section{Statistical Analysis}

In our analyses, we estimated multivariate regression models to assess the effects of distance to the nearest SNC and LEP on access to and use of care.

\section{Study Outcomes}

We used two study outcomes as dependent variables in the regression models (1) having a usual source of care
(USOC), and (2) having at least one physician visit in the prior 12 months. We defined USOC using answers to the question, "Is there a place that you usually go to when you are sick or need advice about your health?" and "What kind of place do you go to most often-a doctor's office, a clinic or hospital clinic, an emergency room, or some other place?" All respondents who indicated having a usual place they go for care, and for whom that place was not an emergency room, were considered as having a USOC. We defined having a physician visit in the past 12 months by the response to the question, "During the past 12 months, how many times have you seen a medical doctor?" Subjects who answered that they had seen a doctor one or more times were classified as having seen a physician in the past 12 months. Of note, due to the wording of the question, the physician visit variable included emergency department visits.

\section{Independent Variables}

We had three primary independent variables of interest: (1) distance from the subject's residence to the nearest SNC, (2) whether the subject had LEP, and (3) their interaction. Thus the models enabled us to assess whether the effects of geographic proximity differ between uninsured people with LEP and those who are English proficient. We determined distance between a subject's residence and nearest SNC from the exact address or nearest cross-street, from which we obtained latitude and longitude, as has been described previously [19]. We used ArcGIS software, version 9.1, to determine the latitude and longitude of the exact address for each SNC and calculated, for each subject, the distance to the nearest SNC. Based on exploratory analyses, we categorized distance as $0-2,2-5$, and 5-10 miles. We determined whether the subject had LEP by subjects' selfclassification of their English proficiency on a 4-point scale (not at all, not well, well, or very well). We defined respondents as having LEP if they reported speaking English less than "well." English-only speakers were not asked this question, but we classified them as speaking English "very well."

We chose covariates in the regression models based on earlier studies of determinants of health care access, most of which use the Anderson Behavioral Model as their underlying conceptual framework [20]. The covariates included sociodemographic characteristics (age, gender, education, marital status, income, race, ethnicity, nativity, number of years since immigration); employment (hours worked weekly); household characteristics (household size, spousal insurance status); health characteristics (overall health status, chronic illness history, cancer history, having mental or emotional health services need); census tract characteristics (suburban community, percent residents 
with LEP, percent residents below federal poverty level); community health care availability (primary care providers per 1,000 residents, distance between residence and nearest emergency department). Spouse insurance status was included as previous studies have shown that family members' utilization of health care predicts an individual's use [21]. We included characteristics of the subject's community because previous studies have shown these to influence health care access [22-26]. All individual-level covariates were derived from CHIS 2005. Primary care physicians per capita in the county was obtained from the 2006 Area Resource File and the community poverty level and prevalence of LEP were obtained from Census 2000. We estimated all models with and without county fixed effects in order to assess whether accounting for unmeasured county-level factors changed our results.

\section{Estimation}

Although the study outcomes are binary, we estimated linear probability models (LPMs), rather than probit or logit models. In an LPM, the dependent variable is binary, but the model is estimated using ordinary least squares regression. We used LPMs because the main focus of our study is on the interaction between LEP and distance. Coefficients of interaction terms in probit and logit models are difficult to interpret and can even be misleading [27, 28], whereas interpretation of interaction terms in LPMs is straightforward. Specifically, in an LPM the coefficient of each independent variable, including interaction terms, is a direct estimate of change in the probability that the outcome variable is one, rather than zero, when that independent variable increases by one unit [25]. Moreover, fixed-effects probit and logit models can be difficult to estimate unless samples are very large. We underscore that LPMs yield consistent estimates (i.e., estimates that are unbiased in large samples) of effect sizes, which is the critical property in our application [22, 29, 30]. All analyses were weighted to reflect the complex design of the CHIS and survey nonresponse.

\section{Results}

\section{Descriptive Data}

Among urban and suburban uninsured Californian adults in $2005,48.5 \%$ had a USOC other than an emergency department. This result differed by English proficiency, with $44.7 \%$ of those with LEP having a USOC, compared to $51.5 \%$ of those who are English proficient $(P=0.005)$. About $55.4 \%$ of urban and suburban nonpregnant uninsured Californian adults had seen a physician at least once in the prior 12 months; this result did not differ significantly by English proficiency (53.6\% of those with LEP compared to $56.8 \%$ of those who are EP, $P=0.201$ ).

The median age for all uninsured adults was 41 years, and about two-fifths were women (Table 1). Additionally, about three-fifths of uninsured adults were Latino, 15\% were Asian, 6\% were African-American, nearly half had never been married, $37 \%$ did not have a high school degree, just over three-fifths were foreign-born, and 30\% were poor. Two-thirds of all uninsured adults lived within 2 miles of the nearest SNC.

Uninsured adults with LEP differed considerably from their English-proficient counterparts in numerous waysthey were slightly older and much more likely to be Latino, be married, lack a high school degree, be foreign-born, and be poor or low-income. Three-fourths of uninsured adults with LEP lived within 2 miles of the nearest SNC, compared with $59.7 \%$ of those who were English proficient $(P<0.001)$.

\section{Regression Results: Usual Source of Care}

As shown in Table 2, uninsured adults with LEP and living within 2 miles of a SNC were $9.3 \%$ less likely than their English-proficient peers to have a USOC $(P=0.04)$. However, the association between distance to the nearest SNC and the probability of having a USOC differed by English proficiency (Table 2). Specifically, increasing distance to the nearest SNC was significantly associated with a lower probability of having a USOC among uninsured adults with LEP. In Model 1a, for example, LEP persons living 5-10 miles from the nearest SNC were $25.2 \%(P=0.003)$ less likely to have a USOC than those living within 2 miles of a SNC and $27.3 \%(P=0.002)$ less likely than persons living between 2 and 5 miles from a SNC. By contrast, distance to the nearest SNC was not associated with having a USOC among EP uninsured adults. Consequently, the difference between LEP and EP uninsured adults in the probability of having a USOC widened as the distance to the nearest SNC increased. Results were similar in models with and without county fixed effects.

\section{Regression Results: Physician Visit}

As shown in Table 3, among uninsured adults living within 2 miles of a SNC there was no difference between LEP and their EP counterparts in the probability of having a physician visit in the prior 12 months. However, as with USOC, increasing distance to the nearest SNC was significantly associated with a lower probability of having a physician visit among uninsured adults with LEP, and the effect size for those living 5-10 miles from the nearest 
Table 1 Characteristics of uninsured non-rural adults in California, by English proficiency, in 2005

(Weighted)
Asterisks indicate that there are statistically significant differences between limited English proficient and English proficient persons with $* P<0.05$, ** $P<0.01$ and $* * * P<0.001$

\begin{tabular}{|c|c|c|c|}
\hline \multirow[t]{2}{*}{ Characteristics } & \multicolumn{3}{|c|}{ Weighted $\% /$ mean } \\
\hline & LEP & $\mathrm{EP}$ & Total \\
\hline Have a usual source of care (other than ER)** & $44.7 \%$ & $51.5 \%$ & $48.5 \%$ \\
\hline Had any physician visit in prior 12 months (including ER) & $53.6 \%$ & $56.8 \%$ & $55.4 \%$ \\
\hline \multicolumn{4}{|l|}{ Distance to between residence and nearest safety net clinic*** } \\
\hline Within 2 miles & $74.0 \%$ & $59.7 \%$ & $66.0 \%$ \\
\hline 2 to within 5 miles & $22.1 \%$ & $32.2 \%$ & $27.8 \%$ \\
\hline 5 to within 10 miles & $3.5 \%$ & $6.1 \%$ & $5.0 \%$ \\
\hline \multicolumn{4}{|l|}{ Age*** } \\
\hline $18-29$ & $29.8 \%$ & $39.6 \%$ & $35.3 \%$ \\
\hline $30-49$ & $51.6 \%$ & $42.2 \%$ & $46.3 \%$ \\
\hline $50-64$ & $17.8 \%$ & $17.6 \%$ & $17.7 \%$ \\
\hline 65 and over & $0.8 \%$ & $0.6 \%$ & $0.7 \%$ \\
\hline \multicolumn{4}{|l|}{ Gender } \\
\hline Female & $43.8 \%$ & $39.5 \%$ & $41.4 \%$ \\
\hline \multicolumn{4}{|l|}{ Race* } \\
\hline Asian/Pacific Islander & $13.8 \%$ & $16.4 \%$ & $15.2 \%$ \\
\hline Black & $1.0 \%$ & $9.1 \%$ & $5.5 \%$ \\
\hline Other/multiracial & $42.9 \%$ & $19.8 \%$ & $30.1 \%$ \\
\hline White & $40.3 \%$ & $49.1 \%$ & $45.2 \%$ \\
\hline American Indian & $2.0 \%$ & $5.6 \%$ & $4.0 \%$ \\
\hline Latino ethnicity*** & $86.0 \%$ & $37.9 \%$ & $59.3 \%$ \\
\hline \multicolumn{4}{|l|}{ Marital status* } \\
\hline Married & $54.7 \%$ & $27.4 \%$ & $39.5 \%$ \\
\hline Divorced/separated/widowed & $11.7 \%$ & $15.0 \%$ & $13.5 \%$ \\
\hline Never married & $33.7 \%$ & $57.6 \%$ & $47.0 \%$ \\
\hline \multicolumn{4}{|l|}{ Educational attainment* } \\
\hline $0-8$ years & $43.7 \%$ & $3.4 \%$ & $21.3 \%$ \\
\hline $9-11$ years & $21.3 \%$ & $11.5 \%$ & $15.8 \%$ \\
\hline 12 or more years & $35.0 \%$ & $85.1 \%$ & $63.8 \%$ \\
\hline Household Size (mean, SD)*** & $4.8(0.1)$ & $3.6(0.1)$ & $4.1(0.1)$ \\
\hline \multicolumn{4}{|l|}{ Immigration status $* * *$} \\
\hline Nonimmigrant & $1.2 \%$ & $64.9 \%$ & $37.0 \%$ \\
\hline Immigrant, in US $<5$ years & $20.5 \%$ & $4.4 \%$ & $11.5 \%$ \\
\hline Immigrant, in US 5 to $<10$ years & $23.9 \%$ & $5.1 \%$ & $13.5 \%$ \\
\hline Immigrant, in US $10+$ years & $54.6 \%$ & $25.8 \%$ & $38.6 \%$ \\
\hline \multicolumn{4}{|l|}{ Family income (\% FPL)*** } \\
\hline$\leq 100 \%$ & $44.8 \%$ & $17.2 \% \%$ & $29.5 \%$ \\
\hline $100-199 \%$ & $41.3 \%$ & $31.4 \%$ & $35.8 \%$ \\
\hline $200-299 \%$ & $6.9 \%$ & $17.8 \%$ & $12.9 \%$ \\
\hline $300 \%+$ & $7.0 \%$ & $33.6 \%$ & $21.8 \%$ \\
\hline Hours of employment, weekly (mean, SD) & $29.6(0.8)$ & $28.5(0.6)$ & $29.0(0.5)$ \\
\hline Married and spouse insured & $17.1 \%$ & $9.2 \%$ & $12.7 \%$ \\
\hline Overall health excellent/very good* & $16.4 \%$ & $46.0 \%$ & $32.9 \%$ \\
\hline 1 or more chronic conditions & $23.0 \%$ & $29.3 \%$ & $26.5 \%$ \\
\hline \multicolumn{4}{|l|}{ Personal cancer history* } \\
\hline None & $98.7 \%$ & $96.3 \%$ & $97.4 \%$ \\
\hline Diagnosis within prior 5 years prior prioryearsyears prior & $0.5 \%$ & $1.2 \%$ & $0.9 \%$ \\
\hline Diagnosis more than 5 years prior & $0.8 \%$ & $2.5 \%$ & $1.7 \%$ \\
\hline Mental health need & $23.6 \%$ & $24.0 \%$ & $23.8 \%$ \\
\hline \multicolumn{4}{|l|}{ Community type } \\
\hline Suburban & $12.2 \%$ & $18.0 \%$ & $15.4 \%$ \\
\hline
\end{tabular}


Table 2 Coefficients of weighted regressions, with distance stratified by English language proficiency, assessing for associations with having a usual source of care

\begin{tabular}{|c|c|c|}
\hline & $\begin{array}{l}\text { Model 1a } \\
\text { No fixed effects } \\
\text { Coefficient (standard error) }\end{array}$ & $\begin{array}{l}\text { Model } 1 \mathrm{~b} \\
\text { Fixed effects } \\
\text { Coefficient (standard error) }\end{array}$ \\
\hline Limited English proficiency & $-0.093 * *(0.045)$ & $-0.091 * *(0.044)$ \\
\hline LEP and distance less than 2 miles & Ref & Ref \\
\hline LEP and distance 2 to $<5$ miles & $0.021^{\mathrm{a}}(0.051)$ & $0.040^{\mathrm{b}}(0.051)$ \\
\hline LEP and distance 5 to $<10$ miles & $-0.252 * * * \mathrm{a}(0.085)$ & $-0.195^{* * \mathrm{~b}}(0.086)$ \\
\hline $\mathrm{EP}$ and distance less than 2 miles & Ref & Ref \\
\hline $\mathrm{EP}$ and distance 2 to $<5$ miles & $-0.050(0.037)$ & $-0.041(0.038)$ \\
\hline $\mathrm{EP}$ and distance 5 to $<10$ miles & $-0.060(0.064)$ & $-0.028(0.065)$ \\
\hline
\end{tabular}

All models are adjusted for covariates, as described in the text

Letters indicate that the two coefficients with matching letters are significantly different, with ${ }^{\mathrm{a}} P<0.01$ and ${ }^{\mathrm{b}} P<0.01$

Asterisks indicate that the coefficient is significantly different from zero or the reference category, with $* P<0.10$, ** $P<0.05$ and $* * * P<0.01$

Table 3 Coefficients of weighted regressions, with distance stratified by English language proficiency, assessing for associations with having had a physician visit in the prior 12 months

\begin{tabular}{lll}
\hline & $\begin{array}{l}\text { Model 2a } \\
\text { No fixed effects } \\
\text { Coefficient (standard error) }\end{array}$ & $\begin{array}{l}\text { Model } 2 \mathrm{~b} \\
\text { Fixed effects } \\
\text { Coefficient (standard error) }\end{array}$ \\
\hline Limited English proficiency & $0.019(0.046)$ & $0.020(0.046)$ \\
LEP and distance less than 2 miles & Ref & $\begin{array}{l}\text { Ref } \\
\text { LEP and distance } 2 \text { to }<5 \text { miles }\end{array}$ \\
LEP and distance 5 to $<10$ miles & $0.050^{\mathrm{a}}(0.051)$ & $0.061^{\mathrm{b}}(0.051)$ \\
EP and distance less than 2 miles & $-0.226^{* * * a \mathrm{a}}(0.087)$ & $-0.222^{* * \mathrm{~b}}(0.087)$ \\
EP and distance 2 to $<5$ miles & Ref & Ref \\
EP and distance 5 to $<10$ miles & $-0.059(0.038)$ & $-0.066^{*}(0.038)$ \\
\hline
\end{tabular}

All models are adjusted for covariates, as described in the text

Letters indicate that the two coefficients with matching letters are significantly different, with ${ }^{\mathrm{a}} P<0.01$ and ${ }^{\mathrm{b}} P<0.01$

Asterisks indicate that the coefficient is significantly different from zero or the reference category, with $* P<0.10$, ** $P<0.05$ and $* * * P<0.01$

SNC was sizable. Among EP uninsured adults, by contrast, the point estimates suggested that longer distance to a SNC might be related to lower likelihood of a visit, but the effect sizes were smaller than that for persons with LEP and failed to reach statistical significance. The result was that the difference between LEP and EP uninsured adults in the probability of having a physician visit widened as the distance to the nearest SNC increased. As with USOC, findings were similar in models with and without county fixed effects.

\section{Discussion}

In this study, we assessed the associations among Englishlanguage prociciency, distance to the nearest SNC, and two frequently used measures of health care access among uninsured adults in California. We found that uninsured adults with LEP were less likely than those who are EP to have a USOC even when they lived within two miles of the nearest SNC. This finding extends what has been previously documented about linguistic disparities in health care access. Although prior studies have shown LEP is negatively associated with health care access among insured and older populations [31, 32], until now this relationship has not been explicitly documented among the uninsured. Our finding is important because it shows that LEP is an additional barrier to having a USOC even in the context of the major access barrier of lacking insurance, By contrast, there was no difference between LEP and EP uninsured adults who lived close to the nearest SNC in the probability of having a physician vists in the previosu year.

Our most important and novel finding, however, is that distance exhibited a significant negative association with 
both having a USOC and having had a physician visit for uninsured people who were LEP, but not for those who were EP. Consequently, differences between LEP and EP adults in health care access widened, in the case of USOC, or first materialized, in the case of having physician visit, with increasing distance to the nearest SNC. Previous studies have not examined whether distance is a greater barrier to access for uninsured persons with LEP than for their EP counterparts.

There are several potential explanations for our findings regarding distance and language proficiency. LEP may be correlated with transportation-related barriers that we could not measure. For example, persons with LEP may have less access to personal transportation or more difficulty navigating public transportation. For these persons, the incremental difficulty associated with traveling a longer distance to a SNC is greater than for English-proficient persons who do not face the same transportation-related barriers.

In a related vein, distance may be a greater barrier for people with LEP because they may need another person, who would need to take time away from other activities, to accompany them to health care visits in order to help them with transportation or provide translation services. The incremental time cost associated with traveling a longer distance is therefore greater for those with LEP, since it is the time for two people, compared to English-proficient persons, who may not need anyone to accompany them. Alternatively, LEP may be associated with cultural predispositions to seek less physician care which, when combined with the burdens of distance, results in less utilization [33].

A potential explanation of a different sort is based on the fact that our analyses did not account for SNCs' linguistic capacities. Thus if LEP persons strongly prefer obtaining care in clinics that offer language-concordant services, our measured distance to the nearest SNC may understate the distance to the nearest relevant SNC-i.e., the nearest SNC that offers such services. Such mismeasurement could lead to overstating the effects of distance relative to an analysis that measured distance to the nearest clinic that offers language-concordant services.

Future studies should explore the relative contributions of these potential explanations, as they have different policy implications. If LEP is correlated with transportation-related access barriers, targeted outreach policies and/ or services are likely needed. Alternatively, if LEP persons mainly need a companion to translate or only SNCs that offer language-concordant services are relevant to them, the distance-based disparities we found may be diminished by expanding translation services and fostering a multilingual health care workforce.

Our finding that among uninsured persons living within two miles of the nearest SNC there were LEP-associated disparities in having a USOC, but not in having a physician visit, is worth highlighting. This finding suggests that, although LEP is likely a barrier to developing a continuity relationship with a provider, it may not be a barrier to obtaining need-based episodic care. Continuity care with a primary care provider is important since people having such are more likely to receive preventive care and less likely to have emergency department visits or be hospitalized [34, 35]. Therefore, policies promoting uninsured persons with LEP developing continuity relationships, such as expanding access to culturally and linguistically-competent primary care, may lead to a decrease in this population's need to access acute care in more costly settings.

Also noteworthy is the diminished association between distance and having a USOC in the models that included county fixed-effects. This finding suggests that unmeasured local factors, including both health care and non-health care infrastructure, are also important. Therefore, it is important to perform local assessments of needs and access barriers when considering policies that influence the structure of the medical safety net.

Our analyses have several limitations that will need to be addressed in future studies. First, our population was limited to the five languages, in addition to English, that are included in the CHIS. Second, low-income and minority populations are less likely to be home owners than low-income whites and to exhibit higher residential mobility [36]. Consequently, the degree of measurement error in the distance variable introduced by residential instability may be different for LEP than English proficient subjects. Third, our clinic database did not include private offices that may not identify themselves as providing free or reduced-priced services, but do provide charity care for a small percentage of their patient populations. Previous studies have shown that, in some communities, such offices are sources of health care [37]. In addition, having a USOC and a physician visit in the prior 12 months are limited measures of health care access and utilization. Further, although our USOC variable excludes emergency departments, we were unable to exclude emergency visits from the physician-visit variable, due to the way the data were collected. Finally, proximity of residence to the nearest SNC is a gross measure of core safety net clinic structure; it does not account for other aspects of SNCs that may influence access, such as the services offered and hours of operation.

Despite these limitations, our study makes an important and novel contribution to the literature on SNCs. Specifically, our finding that among uninsured Californians having LEP is an added barrier to health care access and that the barrier from LEP is compounded when combined with increased distance highlights the multiple ways in which uninsured persons with LEP are disadvantaged in obtaining 
health care. Future studies should explore the mechanisms underlying our findings, so that appropriate potential interventions can be designed and implemented.

Acknowledgments The Robert Wood Johnson Clinical Scholars Program, the Department of Veterans' Affairs, as well as The National Cancer Institute (K07 CA100097) provided funding for this work.

Open Access This article is distributed under the terms of the Creative Commons Attribution Noncommercial License which permits any noncommercial use, distribution, and reproduction in any medium, provided the original author(s) and source are credited.

\section{References}

1. Schoen C, DesRoches C. Uninsured and unstably insured: the importance of continuous insurance coverage. Health Serv Res. 2000;35(1 Pt 2):187-206.

2. Hafner-Eaton C. Physician utilization disparities between the uninsured and insured: comparisons of the chronically ill, acutely ill, and well nonelderly populations. JAMA. 1993;269(6):787-92.

3. Ayanian JZ, Weissman JS, Schneider EC, et al. Unmet health needs of uninsured adults in the United States. JAMA. 2000;284(16):2061-9.

4. Baker DW, Shapiro MF, Schur CL. Health insurance and access to care for symptomatic conditions. Arch Intern Med. 2000;160(9):1269-74.

5. Institute of Medicine. Coverage matters: insurance and health care. Washington: National Academies Press; 2001.

6. Hadley J. Sicker and poorer-the consequences of being uninsured: a review of the research on the relationship between health insurance, medical care use, health, work, and income. Med Care Res Rev. 2003;60:3S-74S.

7. Institute of Medicine. America's health care safety net: intact but endangered. Washington, DC: National Academy Press; 2000.

8. Roby DH, Kominsky GF, Cameron ME. Improving access through health insurance coverage and safety net expansion: a review of the literature. Los Angeles: UCLA Center for Health Policy Research; 2007.

9. Hadley J, Cunningham P. Availability of safety net providers and access to care of uninsured persons. Health Serv Res. 2004;39(5):1527-46.

10. Gresenz CR, Rogowski J, Escarce JJ. Health care markets, the safety net, and utilization of care among the uninsured. Health Serv Res. 2007;42(1):239-64.

11. Probst JC, Laditka SB, Wang JY, Johnson AO. Effects of residence and race on burden of travel for care: cross-sectional analysis of the 2001 US national household travel survey. BMC Health Serv Res. 2007;7:40-53.

12. Flores G, Tomany-Korman SC. The language spoken at home and disparities in medical and dental health, access to care, and use of services in US children. Pediatrics. 2008;121:e1703-14.

13. Derose KP, Baker DW. Limited English proficiency and Latinos' use of physician services. Medical Care Res Rev. 2000;57(1): 76-91.

14. Flores G, Abreu M, Olivar MA, Kastner B. Access barriers to health care for Latino children. Arch Pediatr Adolesc Med. 1998;152:1119-25.

15. Lee S, Brown ER, Grant D, Belin T, Brick JM. Exploring nonresponse bias in a health survey using neighborhood characteristics. Am J Public Health. 2009;99(10):1811-7.
16. Saviano EC, Powers M. California's Safety-Net Clinics: A Primer. November: California Healthcare Foundation; 2005.

17. Rural Health Policy Council. 2006. http://www.ruralhealth.ca. gov/pdf/0502CaliforniaFundedFQHCs.pdf. Accessed 23 May 2006.

18. Rural Health Policy Council. 2006. http://www.ruralhealth. ca.gov/pdf/RHCCALIFONLY-FEB05.pdf. Accessed 23 May 2006

19. Rauch J, Edwards S. CHIS 2005 methodology series: data processing procedures. 2009. Available at: http://www.chis.ucla edu/pdf/CHIS2005_method3.pdf. Accessed 15 Feb 2009.

20. Anderson RM. Revisiting the behavioral model and access to medical care: does it matter? J Health Soc Behav. 1995;36(1): $1-10$.

21. Hanson KL. Is insurance for children enough? The link between parents' and children's health care use revisited. Inquiry. 1998;35(3):294-302.

22. Hargraves LJ, Hadley J. The contribution of insurance coverage and community resources to reducing racial/ethnic disparities in access to care. Health Serv Res. 2003;38(3):809-29.

23. Ponce NA, Gatchell MS, Cordasco KM, Traudt B. Ethnic enclaves, safety net location and cancer screening: Amenity or penalty? International health economics association 6th world congress: explorations in health. Copenhagen, Denmark; 2007. 10 July 2007.

24. Hendryx MS, Ahern MM, Lovrich NP, McCurdy AH. Access to health care and community social capital. Health Serv Res. 2002;37(1):85-101.

25. Aldrich JH, Nelson FD. Linear probability, logit and probit models. California: Sage Publications; 1984. p. 12-9.

26. Pagán JA, Pauly MV. Community-level uninsurance and the unmet medical needs of insured and uninsured adults. Health Serv Res. 2006;41(3 Pt 1):788-803.

27. Ai C, Norton EC. Interaction terms in logit and probit models. Econ Lett. 2003;80(1):123-9.

28. Norton EC, Wang H, Ai C. Computing interaction effects and standard errors in logit and probit models. Stata J. 2004;4(2):154-67.

29. Acs G. Explaining trends in health insurance coverage between 1988 and 1991. Inquiry. 1995;32(1):102-10.

30. Acs G, Danzinger S. Educational attainment, industrial structure, and male earnings through the 1980s. Journal of Human Resources. 1993;26(3):618-48.

31. Ponce NA, Hays R, Cunningham W. Linguistic disparities in health care access and health status among older adults. J Gen Int Med. 2006;21(7):786-91.

32. Pippins JR, Alegría M, Haas JS. Association between language proficiency and the quality of primary care among a national sample of insured Latinos. Med Care. 2007;45:1020-5.

33. Leclere FB, Jensen L, Biddlecom AE. Health care utilization, family context, and adaptation among immigrants to the United States. J Health Soc Behav. 1994;35(4):370-84.

34. Gill JM, Mainous AG, Nsereko M. The effect of continuity of care on emergency department use. Arch Fam Med. 2000;9(4): 333-8.

35. Cabana MD, Jee SH. Does continuity of care improve patient outcomes? J Fam Pract. 2004;53(12):974-80.

36. Gyourko J, Linneman P, Wachter S. Analyzing the relationships among race, wealth, and home ownership in America. J Hous Econ. 1999;8(2):63-89.

37. Fairbrother G, Gusmano MK, Park HL, Scheinmann R. Care for the uninsured in general internists' private offices. Health Aff. 2003;22(6):217-24. 